\title{
Magischer Krankenheiler und Sohn Gottes. DiE JESUANISCHE KRANKENHEILUNG ALS MAGISCHES RituAl UND DAS PhäNOMEN DER DE-Ritualisierung IN DER REDAKTIONELLEN ÜBERLIEFERUNG DER EVANGELIEN Magical Healer and the Son of God. Jesus' Healings as a Magic Ritual and the Phenomenon of De-Ritualization in the Editorial Transmission of the Gospels
}

\author{
AnNika Krahn \\ UNIVERSITY OF COLOGNE \\ akrahn@uni-koeln.de
}

\section{ÜBERSICHT}

Jesus war ein charismatischer Wundertäter. Dies ist allgemein bekannt. Dass Jesus seine Krankenheilungen unter Zuhilfenahme magischer Rituale vollzog - dieses Phänomen wird in der Bibelwissenschaft bislang vernachlässigt. Das liegt mitunter daran, dass Magie im christlichen Kontext mit dubiosen rituellen Praktiken assoziiert wird, die den göttlichen Willen menschlich-magisch beeinflussen.

Eine solche Einschätzung der Magie entspricht unter Berücksichtigung der antiken Quellen jedoch keinen objektivierbaren Kri-

\begin{abstract}
While the depiction of Jesus as a charismatic healer is widely known, his use of magical rituals in connection with those healings is a neglected topic in New Testament exegesis. This may be due to the fact, that in the Christian context magic was soon associated with dubious ritual practices trying to manipulate God's will. The sources, however, offer no objective criteria on the basis of which one could reliably distinguish between charismatic and magical rituals. Instead, the perception of an act as (evil or illegitimate) magic vs. a (good and
\end{abstract}


terien, sondern ist allein Ergebnis kultureller Identitätsarbeit, indem das „fremde“ magisch-rituelle Handeln als illegitime Magie abgewertet wird, wohingegen die „eigenen“ magisch-rituellen Handlungen göttlich autorisiertes Wirken verdeutlichen.

Weil die rituell magischen Krankenheilungen Jesu demzufolge für nicht-christusgläubige Außenstehende mit illegitimen magischen Handlungen verwechselbar waren, trug die redaktionelle De-Ritualisierung der Evangelien dazu bei, unmissverständlich deutlich zu machen, dass Jesus Christus der einzig geborene Sohn Gottes ist. desired) charismatic healing depended entirely on the perspective: While magical rituals performed by Jesus are characterized as charismatic acts because they derived their power from the right authority, similar rituals executed by "others" are devaluated as illegitimate forms of magic. Consequently, one can observe a process of de-ritualization within the synoptic tradition which served to free Jesus from the obscure attribution of being a magician (outsider's point of view) and to establish him as the Messiah and the only-born Son of God (insider's point of view).

\section{SCHLÜSSELWÖRTER}

De-Ritualisierung; Jesus Christus; Krankenheilungen; Magie; Redaktionsgeschichte; Ritual

\section{KeYWORDS}

De-Ritualization; Editorial criticism; Healings; Jesus Christ; Magic; Ritual 
Die Idee Der ERforschung FrÜHChristlicher Rituale hat in der Bibelwissenschaft bereits eine längere Tradition. Vor dem Hintergrund eines historisch-vergleichenden Ansatzes Anfang des 20. Jahrhunderts entstanden Studien, die das Neue Testament mit dem kultischen, liturgischen oder rituellen Kontext der griechisch-römischen Mysterienkulte oder des antiken Judentums in Verbindung brachte. ${ }^{1}$ Diese Forschungsrichtung ist besser bekannt als Religionsgeschichtliche Schule und muss sich zumindest aus heutiger Perspektive („Theologie nach Ausschwitz") die Kritik gefallen lassen, stets die Darstellung des christlichen Supersessionismus zu intendieren.

Diese Beurteilung der Religionsgeschichtlichen Schule sorgte dafür, dass nach dem Zweiten Weltkrieg die Ritualforschung zugunsten sozialgeschichtlicher oder sozialwissenschaftlicher Untersuchungen vernachlässigt wurde bzw. in diesen aufging. ${ }^{2}$ In den folgenden Jahren trat die Ritualforschung besonders dann an prominente Stelle, wenn das Ritual selbst in den Fokus der bibelwissenschaftlichen Forschung rückte, wie beispielsweise die Taufe. ${ }^{3}$ In dem handbuchartigen Werk, Ablution, Initiation, and Baptism, wird deutlich, inwiefern Ritualforschung die Neutestamentliche Wissenschaft bereichern kann, auch wenn sich zugegebenermaßen nur drei Beiträge in dem voluminösen Band des ritualwissenschaftlichen Ansatzes bedienen (u.a. von Strecker und Klostergaard Petersen). Somit kann DeMaris auch 2015 noch eine notorische Abwertung der Ritualforschung seitens der modernen Bibelwissenschaft feststellen, die in Protestantismus und Aufklärung fest verankert ist. ${ }^{4}$ Demzufolge ist eine stetige metakommunikative De-Ritualisierung des Protestantismus zur Kenntnis zu nehmen: „Namentlich der Protestantismus hat im Laufe seiner Geschichte

1. DeMarris, 2015, S. 31-42.

2. Stegemann und Stegemann, 1997.

3. Hellholm et al., 2011.

4. DeMaris, 2015, S. 31. 
stets den Glauben und die innere Gesinnung gegenüber dem Ritual und dem äuBeren Ausdruck bevorzugt". ${ }^{5}$ Parallel zur Gegenüberstellung von Glaube und Ritual wird bis heute zwischen „Gebet" und „magischem Spruch" unterschieden, auch wenn beispielsweise Kremer wenigstens die Möglichkeit fließender Übergänge von einem zum anderen Begriff attestiert. ${ }^{6}$ Diese strenge Kategorisierung führt mit Blick auf die rituell-magischen Elemente der jesuanischen Wundergeschichten in der Bibelwissenschaft zu einer exegetischen Kurz-Sicht: Das Alleinstellungsmerkmal der Krankenheilung des historischen Jesu ${ }^{7}$ sei vor allem die deutliche Reduzierung ritueller Elemente unter Hervorherung seiner charismatischen Fähigkeiten. ${ }^{8}$

In dem vorliegenden Beitrag soll zunächst die objektiv-vergleichbare Kategorisierung von Gebet vs. magischem Spruch in Frage gestellt werden, um auch die Gegenüberstellung von magischen und charismatischen Heilungen zu problematisieren. Infolgedessen können magisch-rituelle Krankenheilungen auch dem historischen Jesus zugerechnet werden, im Rahmen dieser Jesus der magische Ritualaktant ist, der über eine besondere Gottesbeziehung verfügt und dessen Ritual das Heilshandeln Gottes verdeutlicht, indem es Versöhnung ermöglicht und Identität stiftet. Am Ende des Aufsatzes wird die sukzessive De-Ritualisierung der magisch-rituellen Krankenheilungen in der Überlieferung der Evangelien betrachtet. Die Minimierung der magisch-rituellen Elemente brach nicht nur dem von DeMaris beschriebenen Phänomen Bahn, sondern schärfte auch das Profil Jesu als einzig geborenen Sohn Gottes.

\section{Gebet vs. MAgie}

Die von DeMaris aufgezeigte Gegenüberstellung von Glaube und Ritual im Protestantismus und die davon abgeleitete trennscharfe Unterscheidung von Religion und Magie bzw. Gebet und magischem Spruch ist „empirisch nicht haltbar“.9 In manchen Urteilen der Forschung ist zwar in der Unterscheidung das Gebet dadurch bestimmt, dass sich der Mensch der Gottheit unterordnet, an die er sich mit seinen Bitten wendet. Die Erfüllung der Bitten allerdings ist allein der Gottheit vorbehalten. Magie sei im Gegensatz dazu der Versuch, durch rituelle Handlungen mit Zuhilfenahme von Beschwörungsformeln auf die Gottheit einzuwirken und damit letztlich selbst die Erfüllung der eigenen Anliegen durchzusetzen. Dass die Unterscheidung von Magie

\footnotetext{
5. DeMaris, 2015, S. 40.

6. Kremer, 2016, S. 193.

7. Zum Begriff vgl. Wolter, 2019, S. 22.

8. Theißen, 2010, S. 49.

9. Heimbrock, 1994, S. 46.
} 
oder Beschwörung und Gebet des Weiteren mit der Vorstellung einer Kulturentwicklung korrespondiere - also von einer primitiven Kultur zu einer Hochkultur, wie dies beispielsweise Ende des 19. / Anfang des 20. Jahrhunderts der schottische Anthropologe James Frazer behauptete,$-{ }^{10}$ entspricht natürlich nicht dem wissenschaftlichen Status Quo. Überhaupt ist der antiken Welt die idealtypische Trennung von Gebet und Magie/Beschwörung fremd und aus heutiger Perspektive anachronistisch: „Die theoretischen Definitionen von Religion oder Magie haben nur einen geringen praktischen Wert, weil in der Wirklichkeit kultischen Vollzuges sich unsere Begriffe nie voll mit dem tatsächlichen Geschehen zur Deckung bringen lassen ". ${ }^{11}$ Dass außerdem in dem Fall des tatsächlichen Geschehens von Krankenheilungen oder der Abwehr von Bösem ritualisierte Handlungen maßgeblich waren, die im Zusammenhang mit magischen Sprüchen bzw. Gebeten vollzogen wurden, zeigen vier Beispiele aus einem Zeitraum von 2400 (!) Jahren:

Die einzige uns vollständig erhaltene Buchrolle Altägyptens aus dem 16. Jahrhundert v. Chr., das Leipziger Papyrus Ebers, zeugt von einem Rezept ${ }^{12}$ in dem das exsikkierte Auge eines Schweines, echter Bleiglanz, Ocker und „Selbstentstandenes“ von Honig fein zermahlen werden sollen, um dies in das Ohr eines Mannes zu gießen, der von einer Augenkrankheit genesen soll. Dazu soll ein Zauberspruch zweimal wiederholt werden, um die „vortreffliche“ Wirkung zu gewährleisten bzw. überhaupt erst in Gang zu setzen.

Die babylonische Ritualanweisung RA 50 bzw. Or. 34 aus der namburbi-Reihe hat eine apotropäische Funktion. Das Unheil, das sich durch die Mißgeburt eines Tieres oder eines Menschen angekündigt hat, soll abgewehrt werden. Diese Ritualbeschreibung kann in eine Rede- und Handlungsanweisung untergliedert werden, wobei die Beschwörtungstexte bzw. Gebete vom Patienten zu sprechen sind und die Verantwortung für die Ritualhandlung fast ausschließlich beim Magier oder Ritualbeauftragten liegt. ${ }^{13}$ In dem Beschwörungsgebet wird der babylonische Gott Šamaš folgendermaßen angerufen: „Richter des Himmels und der Erde, Herr des Rechts und der Gerechtigkeit, der recht lenkt, was oben und unten ist, Šamaš, den Toten zu beleben, den Gebundenen zu lösen, liegt in deinen Händen. Šamaš, ich bin zu dir gekommen, Šamaš, ich habe dich aufgesucht, Šamaš, ich habe mich an dich gewandt, von dem Bösen dieser Mißgeburt errette mich! Es möge mich nicht erreichen, das

10. Frazer, 2009 (1922).

11. Gerstenberger, 1980, S. 76, vgl. Ricks, 1995.

12. Eb. 356.

13. Gerstenberger, 1980, S. 88. 
Böse davon möge sich entfernen aus meinem Leibe! Täglich will ich zu dir beten, wer mich sieht, möge in Ewigkeit dir huldigen". ${ }^{14}$ Dieses Gebet lässt sich Gerstenberger zufolge sogar in Hymnus, Angebot, Bitte und Lobpreis untergliedern und ist untrennbarer Teil des magischen Rituals. ${ }^{15}$

Aus dem 4./5. Jahrhundert n. Chr. stammt die Quelle P. Köln inv. 10266:

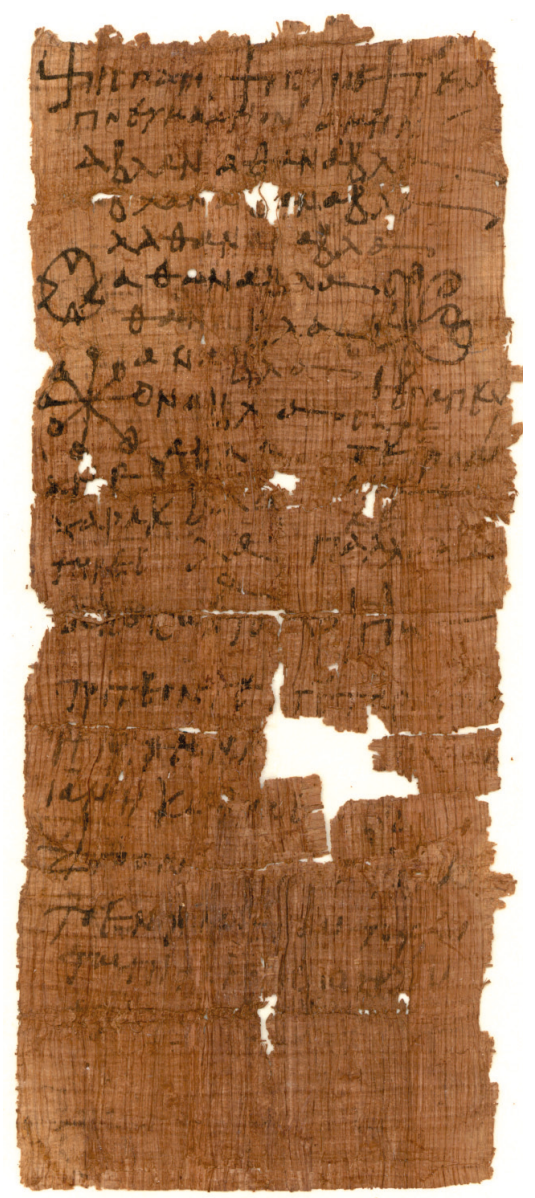

[Swastika] Ein Vater [Swastika] Ein Sohn [Swastika] Ein heiliger Geist. Amen. Ablanathanalba (in Schwindeschrift).

[Heilige Zeichen]

Heile Tiron, den Palladía geboren hat, von allem Zittern, alle drei Tage, alle vier Tage oder jeden anderen Tag oder täglich...

Einer Swastika sprach man apotropäische Bedeutung zu und der Terminus Ablanathanal$b a$ ist das am häufigsten gebrauchte magische Palindrom, ${ }^{16}$ wobei es in Hinblick auf seine Bedeutung noch keine überzeugnde Erklärung gibt. ${ }^{17}$ Unter Berücksichtigung der mit dem Palindrom hier zwanglos verbundenen trinitarischen Anbetung von Vater, Sohn und heiligem Geist ist daher festzuhalten, dass die antiken Quellen eine kategorische Unterscheidung von Gebet und Magie nicht erlauben.

Ein Beispiel aus dem frühen Mittelalter bezeugt ebenfalls die Einheit von Magie/magischem Spruch und Gebet. So ist der Wurmsegen (Pro Nessia), ein oberdeutscher Spruch der Tegernseer Handschrift aus dem 9. Jahrhun-

14. RA 50, übernommen von Gerstenberger, 1980, S. 94-96.

15. Gerstenberger, 1980, S. 96-111.

16. Vgl. PGM III.

17. Vgl. P.Köln 851 (6. Jhd. n. Chr). 
dert, aufgegliedert in eine magische Handlung, respektive einer magischen Beschwörung, der ein dreimaliges Vaterunser folgt: ${ }^{18}$

Gang uz, Nesso, mit niun nessinchilinon

uz fonna marge in deo adra, vonna den adrun in daz fleisk, fonna demu fleiske in daz fel, fonna demo velle in diz tulli.

Ter pater noster

Wurm, krieche heraus, mit neun Würmchen, aus dem Mark in die Adern, aus den Adern in das Fleisch, aus dem Fleisch in die Haut, aus der Haut auf diesen Pfeil.

Dreimal Vaterunser.

Das magische Ritual dient m. E. nicht nur der formellen Unterstützung des Vaterunsers als dem christlichen Hauptgebet, ${ }^{19}$ sondern vielmehr ist das Gebet hier genuiner Teil des magischen Rituals und unabdingbar für die Wirksamkeit der initiierten, imperativisch formulierten Beschwörung. Es wirkt wie dieses nicht automatisch (ex opere operato), sondern ist in seiner Wirkung auf göttliche Hilfe angewiesen. Das Gebet ist somit fest in einen magischen Ritualvollzug eingebunden bzw. geht in ihm auf, und mehr noch, es verhält sich in seiner lateinischen Fassung im Wurmsegen fast wie ein Zauber, der den Fortlauf der Heilung initiiert. ${ }^{20}$

Ist eine strenge Dichotomie zwischen Magie und Gebet im Ritualgeschehen vor dem Hintergrund der gezeigten Beispiele unmöglich, ${ }^{21}$ so gilt dies ebenfalls für Texte aus dem Alten und dem Neuen Testament. ${ }^{22}$ In diesen biblischen magisch-rituellen Heilungsgeschichten können Magie und Religion/Gebet von einem neutralen Beobachter nicht unterschieden werden. Der Versuch einer solchen Differenzierung führe Busch zufolge sogar in eine exegetische Aporie. ${ }^{23}$

Dennoch versucht beispielsweise Gerd Theißen eine solche Differenzierung, indem er in Bezug auf die jesuanischen Wundergeschichten von charismatischen Wundern spricht, die sich ihm zufolge deutlich von den magischen Wundern unterscheiden. Die charismatischen Wunder setzen seiner Meinung nach eine perso-

18. Koch, 1997, S. 42-43.

19. Vgl. Tert., De orat. I 4: Das Vaterunser wird als breviarium totius Evangelii beschrieben.

20. Vgl. Eb. 356.

21. Vgl. PapyCol XVII $1+2$.

22. Z.B. 1 Kön 17,17-27; 2 Kön 20,1-7; Tob 11,7-15; Joh 9,1-7; Apg 5,15; 19,12; Jak 5,14-15.

23. Busch, 2006, S. 14-15.

ARYS, 18, 2020 [281-308] ISSN 1575-166X 
nale Beziehung zwischen Wundertäter und Hilfesuchenden voraus. Diese Art von Wunder seien ohne "Glauben“ oder Vertrauen nicht möglich. ${ }^{24}$ Magische Wunder hingegen benötigen Theißen zufolge keine personale Begegnung zwischen den beiden Protagonisten. Oft geschehen sie somit ohne Wissen und Willen desjenigen, an dem das Wunder vollzogen wird. Darüber hinaus stellt Theißen die magischen und die charismatischen Wunder einander folgendermaßen gegenüber: Die magischen Wunder dienen unabhängig des Gemeinwohls lediglich individuellen Zwecken und oft seien sie auch gegen das Wohlergehen der Gemeinschaft gerichtet. Ferner werden sie durch ritualisierte Praktiken (Beschwörungen, festgelegte Zauberformeln, magische Mittel) vollzogen. Sie sollen ex opere operato wirksam sein. Im Gegensatz dazu ermöglichen allein die charismatischen Wunder Gemeinschaft und geschehen auf Grundlage der Autorität einzelner Wundertäter und mit einem Minimum an ritualisierten Praktiken. ${ }^{25}$ Die charismatischen Wunder Jesu lassen sich zudem eschatologisch deuten, da der Wundercharismatiker Jesus als Heilscharismatiker beschrieben wird, der das Ende der alten und den Beginn einer neuen Welt ankündigt. ${ }^{26}$

Dass die zwei Kategorien der magischen und charismatischen Wunder nicht mehr als eine „idealtypische Vereinfachung" sind, führt Theißen selbst an und führt ferner aus, dass es „sozialer Konvention [unterliege], wo die Grenzen zwischen magischen und charismatischen Wundern gezogen werden. Da diese Grenze umstritten ist, wirkt sich hier in der Tat die Definitionsmacht der Gesellschaft aus: Charismatische Wundertäter können als Magier angegriffen, Magier als charismatische Wundertäter anerkannt werden. So wurde Jesus von seiner Umwelt teils als prophetischer Wundertäter bewundert, teils als Verbündeter des Teufels angegriffen“.27 Dennoch - und das ist die Schwachstelle in der Zuteilung Theißens - attestiert er mit der idealtypischen Unterscheidung einen scheinbar fundamentalen und objektivierbaren Unterschied zwischen magischen und charismatischen Wundern. Die Zuteilung der Verwendungshäufigkeit ritualisierter Praktiken beispielsweise (ein Maximum an Ritualen bei magischen Wundern; ein Minimum an Ritualen bei charismatischen Wundern) ist allein vor dem Hintergrund der metakommunikativen De-Ritualisierung des Protestantismus, ${ }^{28}$ nicht aber auf Grundlage der neutestamentlichen Texte $\mathrm{zu}$ verstehen. Ferner verdeutlicht allein eine kleine Auswahl an neutestamentlichen Perikopen die Schwierigkeit der o.g. idealtypischen Unterscheidung zwischen ma-

24. Vgl. Mk 6,5-6.

25. Theißen und Merz, 2011, S. 277.

26. Theißen und Merz, 2011, S. 279.

27. Theißen und Merz, 2011, S. 277.

28. S.o.; DeMaris, 2015.

ARYS, 18, 2020 [281-308] ISSN 1575-166x 
gischen und charismatischen Wundern: Mt 8,5-13 par Lk 7,1-10 berichtet von der Heilung eines Knechts in Kafarnaum durch Jesus. Der Hauptmann und somit Vorgesetzte des Knechts ist es, der Jesus um dieses Wunder bittet. Die Heilung geschieht demnach mit großer Wahrscheinlichkeit ohne Wissen desjenigen, der geheilt werden soll. ${ }^{29}$ In Mk 11,12-14 par Mt 21,18-19 wird ein Feigenbaum von Jesus verflucht, ewig unfruchtbar zu sein und noch deutlicher gegen das Wohl eines Menschen gerichtet, ist die Blendung eines Magiers durch Paulus, wie sie in Apg 13,8-11 berichtet wird. Auf Grundlage der Quellen, die von diesem mirakulösen Wirken berichten, ${ }^{30}$ ist es in diesem Zusammenhang unwahrscheinlich, dass sowohl umfassende Normen als auch ein theoretisch-terminologisches Konzept von Magie vorlagen, die eine stringente Abgrenzung von Magie zur Religion im frühchristlichen Kontext erlauben (Becker stellt dies für den rabbinischen Kontext heraus). ${ }^{31}$ Otto schlägt auf der Basis seiner weitreichenderen Untersuchungen sogar vor, „auf einen substantiellen Magiebegriff im Wissenschaftsdiskurs" zu verzichten. ${ }^{32}$ Insgesamt scheint demzufolge eine Unterscheidung - sei sie auch noch so idealtypisch - zwischen magischen und charismatischen Wundern wenig tragfähig zu sein. ${ }^{33}$ Mit Bezug auf Weber, der mirakulöses Wirken als einen integralen Bestandteil charismatischer Wundertätigkeit versteht, ${ }^{34}$ muss der Versuch einer Gegenüberstellung von magischen und charismatischen Wundern abgelehnt werden.

Diese Ablehnung einer genaueren inhaltlichen Differenzierung zwischen Magie und Charisma innerhalb der Heilungswunder ist allerdings nicht gleichzusetzen mit Unkenntnis von „Magie“ im frühen Christentum - im Gegenteil. Allein die Simon

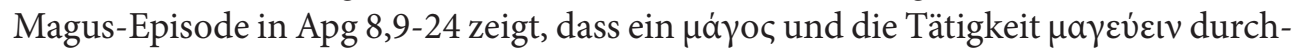
aus bekannt waren. ${ }^{35}$ Jedoch war offenbar der Standpunkt des Betrachters entscheidend dafür, ob ein rituell-magisches Handeln akzeptiert (Binnenperspektive) oder jemand als $\mu \alpha \dot{\alpha} \gamma o \varsigma$ abgewertet oder diffamiert wurde (Außenperspektive). ${ }^{36}$ Unter Berücksichtigung der Begriffe der Selbst- und der Fremdzuschreibung als Aspekte kul-

29. Vgl. ebenso den Exorzismus in Mk 7,24-30 par Mt 15,21-28 und die Auferweckung des Lazarus' in Joh 11,43-44.

30. MkEv, LkEv, MtEv.

31. Becker, 2002, S. 92.

32. Otto, 2009, S. 640.

33. S.o., Heimbrock, 1994, S. 46.

34. Weber, 1982, S. 481.

35. Vgl. Apg 8,9.

36. Vgl. Apg 13,6; explizit von dieser ntl negativen Wertung ausgenommen sind nur die $\mu$ áyol, die in Mt 2, 1-12 in Zusammenhang mit der jesuanischen Kindheitsgeschichte Erwähnung finden; vgl. Tatianus, Ad Gr. 1. 
tureller Identitätsarbeit ${ }^{37}$ - selbstredend unter Annahme eines dialektischen Zusammenspiels des Einen mit dem Anderen ${ }^{38} \mathrm{kann}$ als ein erstes Ergebnis der folgenden Untersuchung Folgendes angenommen werden.

Magie wurde - in der Fremdzuschreibung - als nicht-autorisiertes Ritual angesehen, das in Opposition zu dem vom eigenen Gott autorisierten mirakulösen Wirken stand (Selbstzuschreibung). Ob etwas als Magie (ab-)gewertet wurde, lag also hauptsächlich im Auge des Betrachters, auch wenn es sich um ein und dasselbe mirakulöse Wirken handelte. In Bezug auf die jesuanischen Krankenheilungen bedeutete dies, dass die Deutungshoheit der frühchristlichen Gemeinden - im Rahmen der Selbstund der Fremdzuschreibung - entschied, was nun jeweils magia licita oder magia ilicita war. ${ }^{39}$ Allein die Autorität Gottes legitimierte die magisch-rituellen Handlungen. Jesus ist demzufolge aus der Perspektive des „erinnerten Jesus Christus “ heraus ${ }^{40}$ in der Selbstzuschreibung ein autorisierter Ritualspezialist, dessen magisch-rituelle Handlungen seine Rolle als legitimen Repräsentanten Gottes bzw. Messias verdeutlichen und diese von den mehr oder weniger beteiligten Ohren- und Augenzeugen nicht nur bestätigt, sondern auch als äußerst sinnhaft erlebt werden.

\section{Krankenheilung Als magisches Ritual}

„Das Repertoire der Strukturen magischer Rituale ist begrenzt, und im hohen Maße interkulturell vergleichbar. [...] Zum Ritual gehören eine Vorbereitungsphase (fast immer mit Beachtung asketischer Regeln), eine sich oft zeitlich länger erstreckende Durchführungsphase, die in eine erstrebte Wirkung mündet, und im Falle einer Beschwörung eine förmliche Entlassung des Numens. Wie für die [sic!] meisten Riten sind Anfang und Ende des Ritus meist klar gekennzeichnet. Zum Ritual gehören Worte, Handlungen (Opfer), Gegenstände (die oft im Ritual selbst hergestellt werden), besonderen Zeiten und Orten, spezifische Vorbereitungen sowie allgemeine Rahmenbedingungen. Äußerste Minimierungen sind bestimmte Gesten und Zaubersprüche“. ${ }^{41}$

Schreibt man nun diese Struktur magischer Rituale in ein Aktantengerüst ein, so ergibt sich auf der einen Seite ein Handlungssouverän (Ritualaktant/agent),

37. Brubaker und Cooper, 2000, S. 15.

38. Reed, 2018, S. 407.

39. Vgl. Frankfurter, 2002, S. 439 oder - vor dem Hintergrund der alttestamentliche Sozialgeschichte - Schmitt, 2017, S. 186.

40. Zu dem Begriff vgl. Wolter, 2019, S. 26.

41. Frenschkowski, 2016, S. 101. 
der von einem Adressenten (göttliche Macht) gesendet wurde und positiv auf einen Adressaten (patient) mit Hilfe der rituell durchgeführten Heilung einwirkt - bei einem Schadenszauber könnte man von einer negativen Einwirkung sprechen (action)..$^{42}$ Des Weiteren lassen sich in vielen jesuanischen Krankenheilungsepisoden noch mehr oder weniger unbeteiligte Beobachter finden, deren Aufgabe es ist, den Sachverhalt der Heilung und somit die „Objektivität des wahren Geschehens" zu bezeugen ${ }^{43}$ und diese somit als sinnstiftend zu erleben. ${ }^{44}$

Dies artikuliert sich in der Ritualtheorie von Mary Douglas folgendermaßen: Die handelnden Personen in ihrer Beziehung unter- und zueinander drücken sich ihr zufolge mit Hilfe codierter Sprachformen aus, die diese Art der Beziehungen vermitteln und verstärken, d.h. die Rollen der im Ritual involvierten Personen schärfen. In Anlehung an Basil Bernstein behauptet die Sozialanthropologin, dass dieser Code der Sprachformen mit dem Code der Ritualform korrespondiere und ein Ritual somit eine verdichtete, kohärente Form der Kommunikation darstelle. ${ }^{45}$ Die allgemeine Theorie der rituellen Kompetenz ${ }^{46}$ erweitert das Aktantengerüst und den spachlichen Ritualcode um die unterschiedliche Wirksamkeit der ,", kulturell postulierte[n] übernatürliche[n] Handlungsträger' (Culturally Postulated Superhuman Agents = CPS-Agents) in verschiedenen Teilen des Rituals", wodurch sich Theißen zufolge unterschiedliche Typen religiöser Rituale erkennen lassen. ${ }^{47}$

Ferner sind Rituale und Magie Teil eines Konzepts von dynamischer Reinheit. ${ }^{48}$ Die Befreiung von einem unreinen Geist und die Heilung einer Krankheit vermitteln eine Gruppenidentität, indem sie die bestehende Sozialstruktur wiederherstellen und festigen. ${ }^{49}$ Krankenheilungen bzw. Dämonenaustreibungen enthalten demzufolge ein identitätsstiftendes Moment. Vor diesem Hintergrund bezeichnet Kahl die Heilungen durch Jesus als „all inclusive salvation of God“, die immer wieder gemeinschaftsstiftend seien (Kahl nennt es „communal reintegration“) und die Beziehung zu Gott bestätigen. ${ }^{50}$ Michael Pietsch drückt dies im Zusammenhang mit seiner Untersuchung zu Magie und Ritual in den Elisaerzählungen folgendermaßen aus: „Im per-

\footnotetext{
42. Ebner und Heininger, 2015.

43. Vgl. Mk 7,37 s.u.

44. Vgl. Mk 2,12.

45. Douglas, 1974.

46. McCauley und Lawson, 2002.

47. Theißen, 2017, S. 119.

48. Douglas, 2013.

49. Douglas, 2013.

50. Kahl, 2013, S. 349.
} 
formativen Akt des Ritualgeschehens wird das ordnungsstiftende, heilende Handeln der Götter symbolisch realisiert. Der Beschwörer handelt somit nicht in eigener Vollmacht, sondern als legitimer Repräsentant der Gottheit. Magische Rituale zielen im staatlichen Bereich auf die Sicherung der politischen Ordnung, die in der Person des Königs repräsentiert wird; im familiären Raum dienen sie vorrangig der Abwehr individueller Bedrohungen (z.B. durch Krankheit oder soziale Desintegration). Soziale Antagonismen werden rituell bearbeitet und das gestörte Ordnungsgefüge wieder-

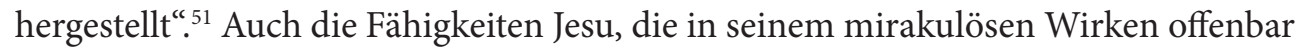
werden, lassen sich in dieses Argumentationsmuster einfügen. Das magisch-rituelle Handeln Jesu ist maßgeblich für die Wiederherstellung der heilvollen Schöpfungsordnung, sodass „Versöhnung zwischen den Menschen“ ermöglicht wird. ${ }^{52}$

Beispielhalber soll an dieser Stelle die Perikope Mk 7,31-37 angeführt werden, in der die Heilung eines Taubstummen beschrieben wird und die als eines von vielen magischen Ritualen der jesuanischen Heilungen gedeutet werden kann:

31 Und als er wieder fortging aus dem Gebiet von Tyrus, kam er durch Sidon an das Galiläische Meer, mitten in das Gebiet der Zehn Städte. 32 Und sie brachten zu ihm

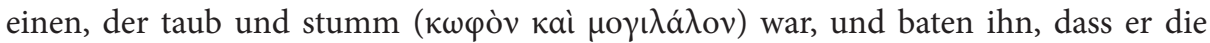

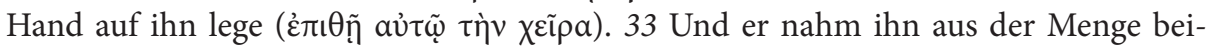

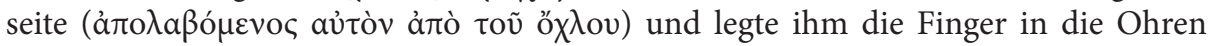

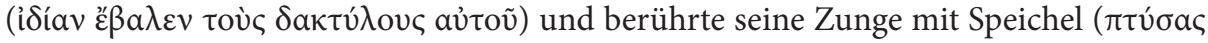

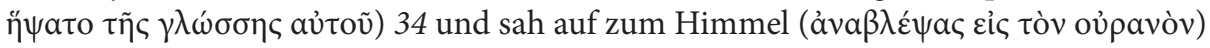

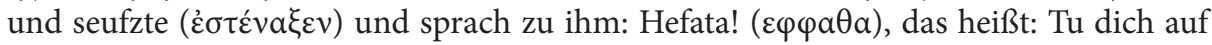

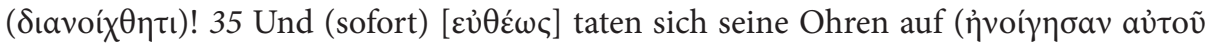

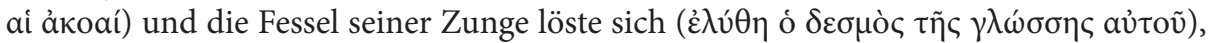

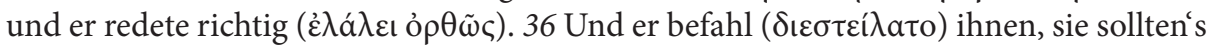
niemandem sagen ( $\mu \eta \delta \varepsilon v i \lambda \hat{\varepsilon} \gamma \omega \sigma i v)$. Je mehr er's aber verbot, desto mehr breiteten sie

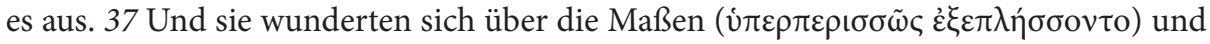
sprachen: Er hat alles wohl gemacht; die Tauben macht er hörend und die Sprachlosen

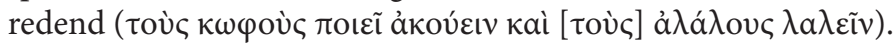

Diese Episode aus dem MkEv zeigt, dass wir im Neuen Testament vergeblich nach Ritualanweisungen nach Art der babylonischen Beschwörungszeremonien suchen..$^{53}$ Im Gegensatz zur Professionalisierung des Berufsstandes der Beschwörungspriester bzw. Magier inkl. spezieller Fachliteratur in Babylon, wie beispielsweise der

51. Pietsch, 2017, S. 345; vgl. Reventlow, 2007; Uro, 2016.

52. Theißen, 2017, S. 128.

53. Vgl. RA 50.

ARYS, 18, 2020 [281-308] ISSN 1575-166x 
mašmašu- und ašipu-Priesterklasse, die ihre Magie wie ein Handwerk gelernt haben, begegnet uns in den Evangelien nur ein Minimum an magisch-rituellen Elementen. „Nevertheless, they are visible and repetitive acts, whose performance is believed to have a physical impact on human beings “. ${ }^{44}$ Trotz der knappen Darstellung des Heilungsritus wird deutlich, dass sich das Wirken Jesu auf die zu heilende Person konzentriert und somit vor dem Hintergrund der Ausführungen von Mary Douglas von einer Beseitigung der sozialen Antagonismen gesprochen werden kann. ${ }^{55}$ Das durchgeführte magische Ritual überwindet dem Konzept der dynamischen Reinheit gemäß das gestörte Sozialgefüge, das in der misslichen Lage des Adressaten deutlich sichtbar wird. Es verdeutlicht zudem die Rettungsmacht Gottes und wirkt somit identitätsstiftend. In der Gestalt Jesu verkörpert sich dieses Rettungshandeln Gottes in der Durchführung und der Ergebnissicherung des magischen Rituals.

Das bereits genannte Rettungshandeln Gottes wird im Rahmen der ritualisierten Krankenheilung vor allem durch eine ansteckende, sich übertragende Kraftwirkung gewährleistet, die in $\mathrm{Mk}$ 7,32 durch das Auflegen der Hände verdeutlicht wird

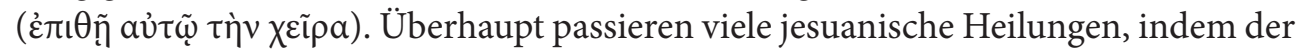
Kranke berührt wird. ${ }^{56}$ Das Auflegen der Hände kann als ansteckendes mirakulöses Wirken gewertet werden. Des Weiteren wird von einer (medizinisch wirksamen?) Paste berichtet, die aus Salvia und Erde gemacht ist; ${ }^{57}$ Das Spucken bzw. der Speichel besitzt eine apotropäische Bedeutung und wird auch unter Berücksichtigung von $\mathrm{Mk}$ 8,23 und vor dem Hintergrund des mirakulösen Handelns des Vespasian mit Hilfe des Auftragens von Speichel, wie in Tacitus' Historien IV 81 und in Suetons De vita caesarum (Titus Flavius Vespasianus) 7, seinen festen Bestandteil in einem (Blinden-) Heilungsritual gehabt haben. Mk 7,35 berichtet davon, dass die Spucke die Kette der

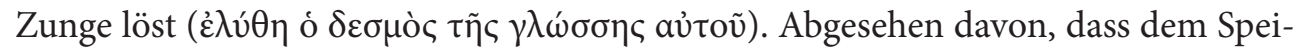
chel in der Antike als besonderer Körpersaft eine apotropäische Wirkung nachgesagt wurde, konnte der Speichel eines anderen Menschen auch Ekel hervorrufen und das Ausspucken verdeutlichte die Verachtung oder Verfluchung eines anderen. ${ }^{58}$ „Die in der Magie (auch der christlichen) wichtige Ekelevokation bzw. Tabuverletzung dient dabei der Willenssteigerung, genauer gesagt dem energischen Verlassen des , Normalen', der Überwindung der, natürlichen' Reaktionen durch reinen Willen [...] “. ${ }^{\text {" }}{ }^{9} \mathrm{Mi}$ -

54. Theißen, 2018, S. 449.

55. Douglas, 2013.

56. U.a. Mk 5,30; 6,56.

57. Mk 7,33; 8,23; vgl. Johannes 9,6-7.

58. Vgl. RGG ${ }^{4}$, s.v. „Speichel“.

59. Frenschkowski, 2016, S. 170. 
rakulöses Wirken ist zudem häufig gekennzeichnet durch die Nennung von Epitheta und eine dem Zauber immanente Hast bzw. Ungeduld: „Jetzt, jetzt, schnell, schnell“ findet als übliche Abschlussformel häufig in den Papyri Graecae Magicae Verwendung. ${ }^{60}$ Diese Beobachtung machte bereits der Kirchenvater Origines. ${ }^{61}$ Im Vergleich mit den von Eile zeugenden Formeln in den PGM kann auch das „sofort" in Mk 7,35 unter Bezugnahmen von u.a. Mk 1,43; 2,12 und 5,42 als eine formelhafte Wendung angesehen werden. ${ }^{62}$ Des Weiteren findet sich in den PGM sehr häufig eine Aufforderung zur Geheimhaltung der Riten als mystifizierende Steigerung von „Privatheit“ und „Bedeutung“.63 Auffallend ist, dass viele jesuanische Heilungsepisoden ebenfalls mit einem Geheimhaltungsgebot abschließen. ${ }^{64}$ In Mk 7,33 wird beschrieben, dass

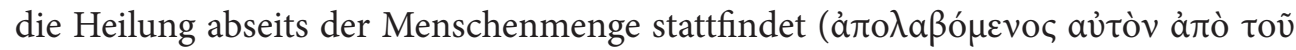
ö $\chi \lambda$ ov). Diese Heilungen im privaten Rahmen, quasi unter vier Augen, wirken, als ob Jesus seine magischen Praktiken zu verstecken versucht. ${ }^{65}$ Auch im außerbiblischen Kontext wird eine ritualisierte Zauberhandlung „außerhalb“, sprich im kultfreien Raum der Natur, meist einfach zu Hause, häufig aber auch im Schlafzimmer vollzogen ${ }^{66}$ Ein weiteres ritualisiertes Element einer Heilung ist das magische Wort ${ }^{67}$ oder das Flüstern bzw. das flüsternde Gebet gen Himmel gerichtet. ${ }^{68}$ In unserem Beispiel Mk 7,34 ist nicht nur der mit Seufzen unterstützte Blick in den Himmel Teil des magischen Rituals, sondern auch das fremdwirkende aramäische „Hefata in Vers 34 dient ähnlich wie das pater noster im Wurmsegen aus dem 9. Jahrhundert als Zauberwort ${ }^{69}$ und könnte als Rhesis barbarike gelten.

Zusammenfassend lassen sich mit Blick auf die antiken, außerbiblischen Quellen bestimmte Merkmale eines magischen Krankenheilungsrituals finden, die auch für das mirakulöse Wirken Jesu bestimmend sind: Die Heilungen finden im privaten Rahmen statt. Die Kranken werden berührt, häufig durch das Auflegen der Hände. Es wird eine Paste verabreicht, gemacht aus Salvia und Erde. Speichel wird verwendet. Ein wichtiges Element ist das magische Wort (Rhesis barbarike) und der Blick in

\footnotetext{
60. Vgl. u.a. PGM XXXV 19; Kotansky, Amulets Nr. 52 in: PapyCol XVII 4; VII 117.

61. Origines Exhortatio ad martyrium 46.

62. Vgl. das عủ $\varepsilon \dot{\omega} \omega \varsigma$ in PGM I 284.

63. PGM I 41; 130; 192; IV 851-857; 1251; 1874; XIII 23; vgl. Plin., N.H. XXX 9 u.a.

64. Mk 7,36: „Und er gebot ihnen, sie sollten's niemandem sagen“, unter Berücksichtigung von u.a.

1,$44 ; 3,9.12 ; 5,43$.

65. Vgl. Mk 5,40; 7,33; 8.23; vgl. Apg 9,40.

66. Ap., Met. IV 20-21; Ps.-Clement., Hom. II 26.

67. „Rhesis barbarike“, vgl. PGM IV 147; vgl. Plin., N.H. XXX 9; vgl. ebenso Lucian., Philops. 9; TSal 7.

68. Uro, 2016; Bremmer, 2017.

69. Vgl. Mk 5,41.
} 
den Himmel. In Bezug auf die Wirkung ist Eile geboten (,jetzt, schnell, sogleich“). Es gibt ein Geheimhaltungsgebot, das befolgt werden muss.

\section{De-RituAlisierung - MiNimiERUNG DER RITUELLENELEMENTE}

Auch wenn streckenweise die vorschriftliche Herkunft der Heilungen Jesu im ca. 70 n. Chr. entstandene Markusevangelium zu erkennen ist, so hat das Jesusbild bereits in der darauffolgenden urchristlichen Überlieferung eine Erweiterung und eine Umbildung erfahren, sodass eine weitere Minimierung der magisch-rituellen Elemente deutlich wird. Bereits das zwischen 80 und $90 \mathrm{n}$. Chr. entstandene Matthäusevangelium drängt magisch-rituelle Elemente redaktionell zurück. So fehlen beispielsweise die markinischen Heilungsgeschichten, in denen der Speichel zum Tragen kommt. ${ }^{70}$ Darüber hinaus werden weitere magische Konnotationen vermieden. Als Beispiel kann eine Krankenheilung angeführt werden, die wahrscheinlich erst im „Nachhinein $\mathrm{zu}[\mathrm{r}]$ Auferweckung[...] von Toten ausgestaltet" wurde. ${ }^{71}$ Es handelt sich um die „Auferweckung der Tochter des Jaïrus“ in Mk 5,35-43 parr. Mt 9,23-26, Lk 8,49-56.

70. Mk 7-31-37; 8,22-26.

71. Wolter, 2019, S. 133. 
Markus 5,35-43

35 Während er noch redete, kommen sie von dem Haus des Synagogenvorstehers und sagen: Deine Tochter ist gestorben, was bemühst du den Lehrer noch?

36 Jesus aber überhörte das Wort, das geredet wurde, und spricht zu dem Synagogenvorsteher: Fürchte dich nicht; glaube nur ( $\mu$ ทे $\varphi \circ \beta o \tilde{\text {, }}$

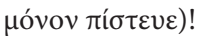

37 Und er erlaubte niemand, ihn zu

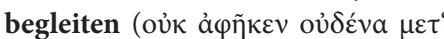

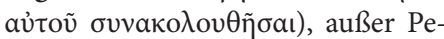
trus und Jakobus und Johannes, dem Bruder des Jakobus.

38 Und sie kommen in das Haus des Synagogenvorstehers, und er sieht ein Getümmel und Weinende und laut Heulende.

39 Und er geht hinein und sagt zu ihnen: Was lärmt und weint ihr? Das Kind ist nicht gestorben, sondern

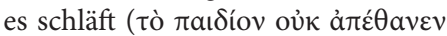
à $\lambda \lambda \grave{\alpha} \kappa a \theta \varepsilon v ́ \delta \varepsilon \iota)$.

40 Und sie lachten ihn aus. Als er aber alle hinausgetrieben

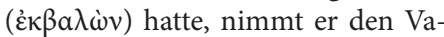
ter des Kindes und die Mutter und die, die bei ihm waren, mit und geht hinein, wo das Kind war. 41 Und er ergriff die Hand des Kindes

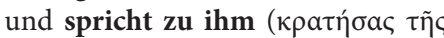

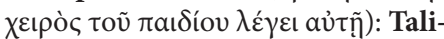

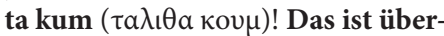
setzt: Mädchen, ich sage dir, steh

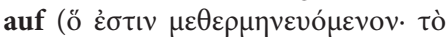

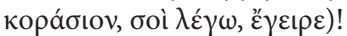

42 Und sogleich ( $\varepsilon \dot{v} \theta \dot{v} \varsigma)$ stand das Mädchen auf und ging umher

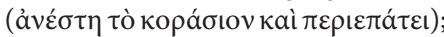
es war nämlich zwölf Jahre alt. Und sie erstaunten (sogleich) mit großem Erstaunen.

43 Und er gebot ihnen dringend, dass niemand dies erfahren sol-

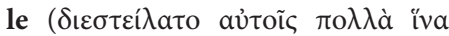

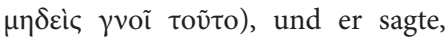
man solle ihr zu essen geben.
Matthäus 9,23-26

23 Und als Jesus in das Haus des Vorstehers kam und die Pfeifer und die lärmende Volksmenge sah,

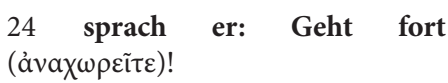

Denn das Mädchen ist nicht gestorben, sondern es schläft.

Und sie lachten ihn aus. 25 Als aber die Volksmenge hinausgetrieben war,

ging er hinein und ergriff sie bei der Hand; und das Mädchen stand auf

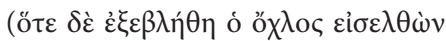

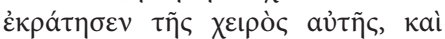

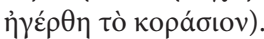

26 Und die Kunde hiervon ging hinaus in jene ganze Gegend.
Lukas 8,49-56

49 Während er noch redete, kommt einer von dem Haus des Synagogenvorstehers und sagt zu ihm: Deine Tochter ist gestorben. Bemühe den Lehrer nicht!

50 Als aber Jesus es hörte, antwortete er ihm: Fürchte dich nicht, glaube

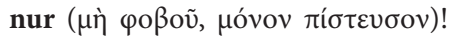
Und sie wird gerettet werden.

51 Als er aber in das Haus kam, erlaubte er niemand hineinzugehen

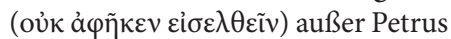
und Johannes und Jakobus und dem Vater des Kindes und der Mutter. 52 Alle aber weinten und beklagten sie.

Er aber sprach: Weint nicht! Denn sie ist nicht gestorben, sondern sie schläft.

53 Und sie lachten ihn aus, da sie wussten, dass sie gestorben war.

54 Er aber ergriff ihre Hand und

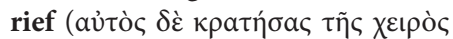
aủंก̃ऽ $\dot{\varepsilon} \varphi \omega ́ v \eta \sigma \varepsilon v)$

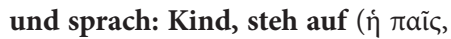
हैүع

55 Und ihr Geist kehrte zurück, und sogleich stand sie auf;

und er befahl, ihr zu essen zu geben

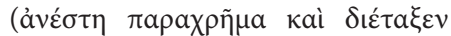

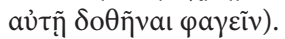

56 Und ihre Eltern gerieten außer sich; er aber gebot ihnen, niemand zu sagen, was geschehen war $(\dot{o} \delta \grave{\varepsilon}$

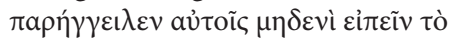
үeүovós). 
Diese Erzählung lässt besonders im MkEv Züge eines Rituals erkennen, an dem als Akteure der Adressat/patient (in diesem Fall die Tote) sowie ein Ritualaktant/ agent (Jesus) beteiligt sind. Darüber hinaus wird ein Synagogenvorsteher genannt, der den Gottesmann beauftragt. Das Ritual findet bei dem Kranken (hier: der Toten) zu Hause statt. Wesentlich ist, dass Jesus in Mk 5,40 die Heilung (Erweckung) in einem privaten Rahmen stattfinden lässt, ein quasi-magisches Wort spricht und die Tote berührt. Zugleich wird ein Geheimhaltungsgebot ausgesprochen, dass die Eltern befolgen sollen, ${ }^{72}$ die zugleich Ohren- und Augenzeugen der magisch-rituellen Heilung sind. Ferner lässt sich die im Zauber typische Ungeduld finden. ${ }^{73}$ Dass das Mädchen wieder lebt, erkennt man an ihrem Umhergehen. ${ }^{74}$ Eine Art der öffentli-

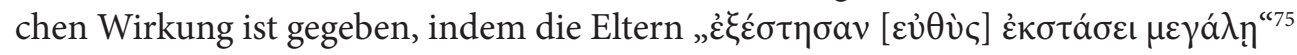
und ihrer Tochter zu essen geben sollen. ${ }^{76}$ Gerade letzteres ist immanenter Teil des Heilungsprozesses, denn eine Krankheit sowie der Tod isolieren aus der menschlichen Gemeinschaft. Zur Heilung gehört hingegen, dass eine Wiederaufnahme in die Gemeinschaft stattfinden kann. ${ }^{77}$

Wenn Markus in 5,41 die gr. Übersetzung neben das aramäische „Zauberwort“, "Talita kum", stellt und somit die magisch klingende Fremdartigkeit dieser Wörter zumindest zulässt, so lässt der Evangelist Matthäus die wörtliche Rede komplett weg und berichtet nur von der körperlichen Berührung. ${ }^{78}$ Lukas übernimmt allein die griechische Übersetzung, „Kind, steh auf!“ ${ }^{79}$ Diese Art der De-Ritualisierung ist auf die Redaktion der Evangelisten zurückzuführen. Das Zurücktreten der ausführlicheren Beschreibung von Krankenheilungen zeigt: Der magisch-rituelle Aspekt des Wunderwirkens Jesu hat auch die Christen in Verlegenheit gebracht. Das apokryphe ThEv aus dem 2. Jhd. n. Chr. beispielsweise weiß von überhaupt keinen Heilungswundern Jesu zu berichten. Die apokryphen ActAp (2./3. Jhd. n. Chr.) nennen zwar viele mirakulöse Heilungsgeschichten. Diese sind allerdings - wie der Name schon sagt - allesamt auf die Apostel Jesu beschränkt und vor dem Hintergrund hagiographischer Literatur zu verstehen. Vor dem Hintergrund der Selbst- und der Fremd-

\footnotetext{
72. $\mathrm{Mk} 5,43$.

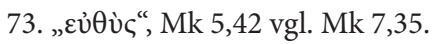

74. $\mathrm{Mk} 5,42$.

75. $\mathrm{Mk} 5,42$.

76. $\mathrm{Mk} 5,43$.

77. Douglas, 2013.

78. Mt 9,25 .

79. Lk 8,54 .
}

ARYS, 18, 2020 [281-308] ISSN 1575-166X 
zuschreibung kann allerdings auch in den ActAp zwischen magia licita oder magia ilicita unterschieden werden. ${ }^{80}$

Beim Apostel Paulus als Verfasser der ältesten uns heute noch erhaltenen Schriften des Neuen Testaments fehlen des Weiteren direkte Hinweise auf die Heilungswunder Jesu, abgesehen davon, dass er in 2 Kor 12,8 berichtet, den Herrn von eigener Krankheit gequält selbst dreimal angerufen zu haben (im Kontext wird deutlich, dass es in 2 Kor 12,7-9 um die Bitte des kranken Paulus geht, den Satansengel abzurufen). Dass die jesuanischen Wunder bei ihm überhaupt keine Erwähnung finden, liegt daran, dass die Grundlage seines Glaubens die Begegnung mit dem auferstandenen Christus war. Von hier aus begründet er seine Theologie. Das heißt allerdings nicht, dass er keine Wunder Jesu kannte und/oder er einen wunderfreien Jesusglauben vertrat. Der Apostel kennt das Charisma der Heilung sehr wohl ${ }^{81}$ und behauptet selbst, Zeichen, Wunder und Machttaten in Korinth gewirkt zu haben. ${ }^{82}$

Die Tendenz der synoptischen Evangelien der De-Ritualisierung kann demzufolge sachlogisch mit dem Sitz im Leben der Evangelien begründet werden: Es zeigt die apologetische Färbung der Evangelien. Beurteilt man die Evangelien in Analogie zu alttestamentlichen Propheten-Biographien (Jer, DtJes), ${ }^{83}$ antiken Biographien wie der Alexander-Vita des Plutarchs oder dem Alexanderroman, so wird deutlich, dass die Evangelisten den Gottessohn Jesu Christi vor dem Vorwurf zwielichtiger Magie (als Fremdzuschreibung) zu schützen versuchen und aus diesem Grund die magisch-rituellen Elemente reduzieren ${ }^{84}$ Sind die magischen Rituale dadurch gekennzeichnet, dass sowohl die wirkmächtige Handlung als auch das wirkmächtige Wort zusammengedacht werden müssen, so verlagert sich in der urchristlichen Tradition der Schwerpunkt auf das wirkmächtige Wort. Besonders deutlich zeigt sich dies im MtEv, in dem Jesus vor allem als Messias des Wortes vorgestellt wird. Als Sohn Gottes, der Auferstandene, der Erhöhte und Herr über alle dämonischen Mächte ${ }^{85}$ wird Jesus zudem im Rahmen der Überlieferung von dem Vor-Urteil eines unseriösen Magiers befreit und der Glaube wird in den Mittelpunkt gerückt. Vergleichbar mit der Aussage Jesajas in Jes 7,9, „Glaubt ihr nicht, dann bleibt ihr nicht“, konzentrieren sich die jesuanischen Krankenheilungen auf die Wirkmächtigkeit des Glaubens.

80. Bremmer, 2017; vgl. Reimer, 2002.

81. 1 Kor 12,9.

82. 2 Kor 12,12 .

83. Vgl. Heil, 2009, S. 89.

84. Uro, 2016.

85. Mt 28,18; Phil 2,6-11. 
Ohne Analogie in den antiken Quellen ist der Zuspruch, „Dein Glaube hat Dir geholfen! ${ }^{\text {"86 }}$ Berger führt derlei Aussagen über den Glauben auf eine Teilhabe an Gottes Kraft zurück und ordnet diese der "technischen Missionssprache“ $\mathrm{zu}^{87}$

Auch das nach der Zerstörung des zweiten Tempels ab $70 \mathrm{n}$. Chr. sich entwickelnde rabbinische Judentum verfährt ähnlich, denn auch dort werden Beschreibungen magisch-ritueller Praktiken zurückgedrängt. Durch Anwendung magischer Techniken (z.B. das Zeichnen eines Kreises) vollbringt Choni im 1. Jhd. v. Chr. beispielsweise ein Regenwunder. Die Brisanz der magischen Handlung von Choni, der auch Kreiszieher genannt wurde, wird mit der Erfüllung von Hab 2,1 als Schriftbeweis im Talmud entschärft. ${ }^{88}$ Dieser zunehmende Rabbinisierungsprozess befreite Choni von Vorwürfen der Magie und integrierte den Kreiszieher in die Rechtgläubigkeit. „Aus dem umstrittenen Magier ist ein anerkannter Rabbi geworden“.89

Ein weiterer Wundertäter in der Umwelt Jesu ist Chanina ben Dosa. ${ }^{90}$ Er lebte in der Mitte des 1. Jhd. n. Chr. in selbst gewählter Besitzlosigkeit und wurde als Sohn Gottes angesehen. Im Talmud sind seine Wunder erzählerisch entfaltet und es wird berichtet, dass es Chanina möglich war, die Lebenschancen schwer erkrankter Menschen hervorzusagen, die um Heilung baten und Gott anflehten. Auch wird über die Heilung des Sohnes Gamaliels berichtet. ${ }^{91}$ Im Talmud wird allerdings deutlich gemacht, dass Gott als Adressant durch seinen Handlungssouverän (Chanina) die Wunder bewirkt. Ben Dosas Wunderkraft wird auf seine intensive Gottesbeziehung zurückgeführt und die (typologischen) Verweise auf die großen Wunderpropheten des Alten Testaments (Elia/Elisa) verfolgen die Absicht, ihn in ebendieser Traditionslinie zu verorten, sodass er keinesfalls als windiger Magier wahrgenommen werden kann.

Ein letzter Vergleich birgt eine interessante Parallele zur Verfluchung des Feigenbaumes durch Jesus (Mk 11,12-25). In einer Erzählung des Talmuds (bTaan 24a) bespricht ein Sohn Rabbis Joses einen Feigenbaum in direkter Anrede, damit die armen Arbeiter von den Früchten des Baumes essen können. Diese magische Handlung wird als illegitime Belästigung des Schöpfers beurteilt und endet in der Verfluchung des Sohnes, auch wenn dieser es nur gut gemeint hat.

86. Vgl. u.a. Mk 5,34.36; 9,23.

87. Berger, 1995, S. 387 und 631.

88. mTaan 3,8 / bTaan 23a.

89. Kollmann, 2002, S. 45.

90. bBer 34b / jBer 5,5 / bBer 34b.

91. Vgl. Mk 5,35-43. 
Die Magie sollte entschieden von dem Bereich der rechtmäßigen Religion im rabbinischen Judentum ausgeklammert werden, damit die Macht Gottes nicht in Frage gestellt werden kann. Jesus Christus als Sohn Gottes verdeutlicht diese Macht in urchristlicher Tradition. „(The) experience of divine empowerment (coupled with a special sense of a close relationship with God) prompted Jesus to reinterpret, combine and transform [...] traditions. He then understood his individual acts of healing and exorcism as prophetic signs, not only of the imminence of the Kingdom of God, but of its nature". 92

Vor dem Hintergrund des Gesagten wird nochmals deutlich, dass die Unterscheidung von unautorisierter Magie und autorisiertem magischen Ritual im Zusammenhang mit Identifikations- und Abgrenzungsprozessen (Selbst- und Fremdzuschreibung) Bestand hat. „[S]owohl im Ausgrenzungs- als auch im Aufwertungsdiskurs war der Magiebegriff [...] weniger durch die Indikation semantischer Bedeutung, als vielmehr durch Funktionen der Aus- und Eingrenzung, der $\mathrm{Ab}$ - und Aufwertung eigener und fremder Identität(en) in einem hochkomplexen, interreligiösen Diskursfeld gekennzeichnet. Entscheidend ist: diese Strategien - Bewertung; Abgrenzung; Identitätsstiftung - stellen Funktionalisierungen von Sprache dar, die in religiösen Diskursen eine gewichtige Rolle spielen mögen, im religionswissenschaftlichen Diskurs aber (idealerweise) vernachlässigt, wenn nicht ganz ausgeblendet werden sollten“. ${ }^{\text {" }}{ }^{3}$ Die Verwendung des Magie-Begriffs ist ein polemisch-identitätsstiftendes Sprachspiel und im Rahmen der frühchristlichen Gemeinden als Fremdzuschreibung „a set of beliefs and practices that deviated sharply from the norms of the dominant social group, and was thus considered antisocial, illegal, or unacceptable “.94 Kurz und bündig gesagt: ",[M]agic' $[\ldots]$ is quintessentially the activity of the ,outsider ' in the Bible ${ }^{\text {" } 95}$

Dtn 18,9-14 stellt eine Liste an Handlungen heraus, die als Magie bzw. Zauberei disqualifiziert werden und infolgedessen ein Gegenüber von autorisiertem und nicht autorisiertem magischen Wirken markieren. Bereits in der Rezeption dieses Textes in der Qumran-Literatur in 11Q19 wird deutlich, dass es um die Bestimmung einer kultischen Konformität geht, die es einzuhalten gilt. ${ }^{96}$ Auch die Weisheit Salo-

92. Eve, 2002, S. 381.

93. Otto, 2009, S. 641.

94. Ricks, 1995, S. 131. Vgl. aus außerchristlicher Perspektive Plin., N.H. XXX 15; Ap., De Magia, passim; Lucian., Philops. IX 16.

95. Ricks, 1995, S. 132.

96. Paganini, 2017. 
mos postuliert einen Gegensatz von Magie bzw. Zauberei und Glaube. Magie sei ein "abscheuliches Verbrechen ${ }^{\text {“97 }}{ }^{97}$ Und auch im Neuen Testament finden sich derartige Spuren von Magiepolemik, ${ }^{98}$ die ebenfalls in der frühchristlichen Didache (ca. 100 n. Chr.) ihren Niederschlag finden: u.a. Magie ( $\mu \alpha \gamma \varepsilon \tilde{\imath} \alpha)$, Götzendienste und Giftmi-

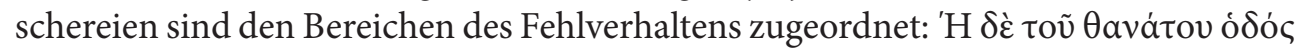

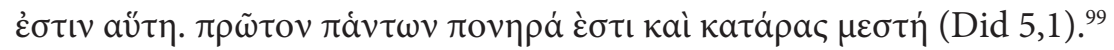

\section{War Jesus ein Magier?}

Zweifellos war der historische Jesus ein Heiler und ein Exorzist, der die Naturgesetze durchbrach. ${ }^{100} \mathrm{Ob}$ Jesus allerdings ein Magier genannt werden kann oder nicht, ist m. E. mit Blick auf die Evangelien allein eine Sache des Standpunktes und es kommt darauf an, ob die Unterscheidung, die man vornehmen möchte, aus der Binnenperspektive (Selbstzuschreibung) heraus getroffen oder als Fremdzuschreibung durchgeführt wird. ${ }^{101}$ Diese Art der Differenzierung macht deutlich, dass beispielsweise in frühjüdischer Deutung entweder die Macht Gottes oder z.B. bei ägyptischen Magiern eine nicht autorisierte Macht am Werk war. ${ }^{102}$ Die frühchristliche Rechtfertigung der Fähigkeiten Jesu orientiert sich an diesem Argumentationsmuster und so sahen die christusgläubigen Gemeinden Jesus als legitimen Repräsentanten Gottes, respektive Sohn Gottes. „Jews and pagans, on the other hand, who viewed the Christians as the, outsiders', viewed Jesus' miraculous acts as either fraudulent or demon-inspired, but in either case , magical '“. ${ }^{103}$ Spätere antichristliche Polemiken schildern, ${ }^{104}$ dass Jesus ein Volksverführer und ein Zauberer gewesen sei. ${ }^{105}$ In der rabbinischen Literatur berichtet bSanh. 43a von dem Zauberer Jesu, der das Volk Israel verführt habe. Auch in der antichristlichen Polemik der sagengeschichtlichen Toledot Jeschu wird Jesus als Zauberer und Volksverführer beschrieben, der die Magie in Ägypten gelernt habe. Unsicherheiten bestehen in

\footnotetext{
97. SapSal 12,4, vgl. 18,13.

98. Vgl. Apg 8,9; 13,6; 16,16-18; 18,23; 19,19; Gal 5,20.

99. Vgl. in diesem Zusammenhang auch Mastrocinque, 2005, S. 216.

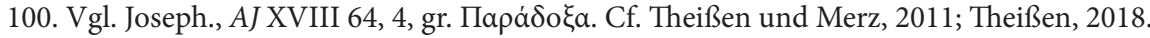

101. Ricks, 1995.

102. Vgl. Joseph., BJ VII 180, 5. Cf. Eve, 2002; Swartz, 2018.

103. Ricks, 1995, S. 142.

104. Z.B. die des Celsus vgl. Origen, C. Cels. I 29; II 32, 49; VIII 39, 41.

105. Vgl. Justin, Apol. XXX 1.
} 
der Bestimmung der Entstehungszeit der Sagensammlung. So wird vermutet, dass Fragmente der Toledot Jeschu bereits im 3.-5. Jhd. entstanden sind. ${ }^{106}$

Dass allerdings bereits dem irdischen Jesu Magie und Zauberei nachgesagt wurden, zeigt das MkEv:

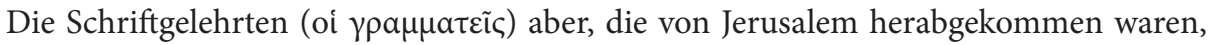
sprachen: Er hat den Beelzebul, und: Er treibt die bösen Geister aus durch ihren Obers-

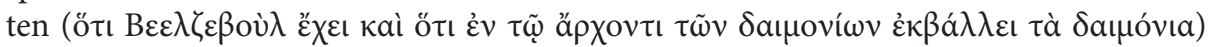
(Mk 3,22).

Die Gegner Jesu zweifeln nicht daran, „dass er Dämonen austreibt, sondern werfen ihm lediglich vor, dass er sich dabei die Hilfe einer stärkeren dämonischen Macht zunutze macht“ ${ }^{\text {“ }} 107$ Jesus wird des Gebrauches dämonischer Mächte verdächtigt und somit implizit der Magie. ${ }^{108}$ Den o.g. Untersuchungen gemäß gelten die Schriftgelehrten als Außenstehende bzw. Gegner, denen das Wirken Jesu unbekannt, wenn nicht sogar ungeheuerlich war. Auch die Verse in Mk 6,14-15 und 8,27-28 verdeutlichen, dass die Unbekanntheit Jesu einer alte Traditionsstufe entspricht.

Und Jesus und seine Jünger gingen hinaus in die Dörfer von Cäsarea Philippi. Und auf dem Weg fragte er seine Jünger und sprach zu ihnen: Was sagen die Menschen, wer

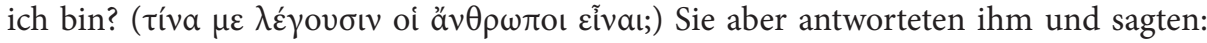

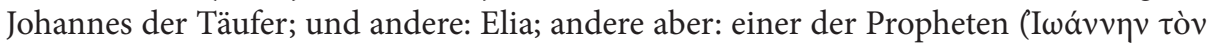

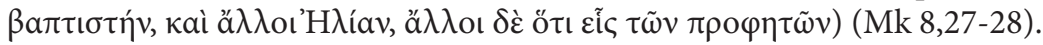

Seine Widersacher sagen, er sei besessen ${ }^{109}$ oder als im Bund mit dem Teufel stehend. ${ }^{110}$ Zudem passt der Versuch, ihn als Elia redivivus zu deuten, nicht in das nachösterliche Bild von ihm, denn aus christlicher Perspektive ist nicht Jesus, sondern Johannes der Täufer der Elia redivivus. ${ }^{111}$ Es gibt daher keinen Grund zu zweifeln, dass auch Nicht-Anhänger von seinen magisch-rituellen Wundern irritiert oder zumindest beeindruckt waren, die für sie in einer Fremdzuschreibung als Magie gedeutet wurden, deren wirkmächtige Instanz wiederum unbekannt oder illegitim war. Insgesamt kann also zum einen festgestellt werden, dass die Überlieferung der Hei-

106. Meerson und Schäfer, 2014.

107. Wolter, 2019, S. 131.

108. Ricks, 1995, S. 140.

109. Mk 3,20-22 parr.

110. Vgl. Lk 11,14-23.

111. Mk 9,13; Mt 11,14. 
lungen nicht auf seine Anhänger und auf die Christusglaubenden beschränkt war. Zum anderen ist festzuhalten, dass Jesus aus frühchristlicher Perspektive (Selbstzuschreibung) kein Magier war, ${ }^{112}$ seine Heilungen aber für Gegner und Außenstehende mit negativ konnotierter Magie und Verführungszauber ${ }^{113}$ aus dem Machtbereich der Dämonen in Verbindung gebracht wurden (Fremdzuschreibung). Auch den Evangelisten fiel diese Verwechselbarkeit auf und so lässt Mk Jesus auf die Anschuldigung in 3,22 im Sinne der De-Ritualisierung allein sachliche Gründe anführen, die den Vorwurf widerlegen: Die Einheit des Machtbereiches des höchsten Dämons bzw. des Teufels würde zerbrechen, wenn sich seine Angehörigen selbst bekämpfen würden. ${ }^{114}$ Ein gegen sich selbst kämpfender Teufel ist reine Makulatur. Im Endeffekt besitzt Jesus also nicht nur die Vollmacht Gottes als Legitimationsgrundlage seiner Handlungen, sondern auch die besseren Argumente; ${ }^{115}$ interessante Abgrenzungsmöglichkeiten ergeben sich in diesem Zusammenhang z.B. mit den apokryphen Evangelien und den apokryphen Apostelakten. ${ }^{116}$

\section{FAzit}

Wenn Samson Eitrem 1966 behauptet, dass Jesus lediglich in Momenten der spirituellen Schwachheit von Techniken der magischen Volksmedizin Gebrauch gemacht habe, ${ }^{117}$ oder Morton Smith 1978 darüber hinausgehend versucht, Jesus in vollem Umfang als Magier darzustellen, ${ }^{118}$ „,dessen religionsgeschichtlicher Rahmen weniger durch das zeitgenössische Judentum als vielmehr durch die antiken Wandermagier beschreibbar ist", ${ }^{\prime 19}$ dann unterläuft den beiden der Fehler, in Bezug auf den Magiebegriff nicht zwischen Selbstzuschreibung und Fremdzuschreibung zu unterscheiden und infolgedessen eine anachronistische Sicht zu postulieren. Jesus war selbstredend kein Magier und er war es zweifelsohne doch. Entscheidend ist allein die Perspektive, von der aus man darüber urteilt. Objektivere Kriterien, die eine Differenzierung zwischen magischen und nicht-magischen bzw. charismatischen Wundern ermöglichen, sind anachronistisch und führen zwangsläufig in eine Aphasie. In der Selbstzuschreibung wird die Rolle Jesu durch die magisch-ritualisierten Krankenheilungen als au-

112. Vgl. Mk 1,22.

113. Vgl. Apg 13,6.

114. Wolter, 2019, S. 144.

115. Vgl. Mt 4.

116. Reimer, 2002; Bremmer, 2017.

117. Eitrem, 1966.

118. Smith, 1978.

119. Busch, 2006, S. 98. 
torisierter Mittler geschärft, dessen spezielle Gottesbeziehung sichtbar und erlebbar wird. Das jesuanische Handeln erzeugt für die (glaubenden) Beobachter Sinn und wirkt identitätsstiftend, indem es eine Notsituation überwindet, Versöhnung ermöglicht und somit die heilvolle Schöpfungsordnung wiederherstellt. „Wenn Jesus den Menschen Heilung bringt, bringt er ihnen das Heil Gottes. Wenn Jesus Menschen heilt, schenkt er ihnen, was nur Gott schenken kann". ${ }^{120}$

Die Wiederherstellung der Schöpfungsordnung ist in diesem Zusammenhang ein Bestandteil der Installation der Gottesherrschaft. Jesus selbst ist es auch, der seine durch ihn und durch den Glauben der Adressaten gewirkten Wunder als Gleichnishandlung für den Anbruch des Gottesreiches versteht. ${ }^{121}$ Es ist demnach allein der Ritualaktant Jesus, „durch den die Königsherrschaft Gottes zu den Menschen kommt, und [...] der die eschatologische Hoffnung erfüllt die sich bisher in exklusiver Weise auf Gott richtete “. ${ }^{122}$ Mit der Redaktion der Evangelisten und der Reduktion der magisch-rituellen Elemente tritt diese Rolle Jesu aus nachösterlicher Perspektive immer deutlicher hervor. Die Evangelien profilieren das Jesusbild, indem sie eine Jesus-Biographie aus einer konsequent nachösterlichen Glaubensperspektive heraus formulieren und demzufolge die theologische Absicht vertreten, die Vollmacht und den Auftrag Gottes der Botschaft Jesu zugrunde zu legen. Die Evangelien sind also keine Erzählungen über Jesu allein, sondern auch ein theologisches Statement. ${ }^{123}$ In diesem Zusammenhang findet auch im Rahmen der synoptischen Tradition eine konsequente Abgrenzung zur Magie als Fremdzuschreibung statt: Insbesondere das MtEv und das LkEv versuchen, die dubiosen magisch-ritualisierten Elemente zu minimieren, um Jesu vom Vorwurf der halbseidenen Magie zu befreien. ${ }^{24}$ Die De-Ritualisierung ist das Ergebnis eines solchen Differenzierungsprozesses. Jesus Christus gewinnt als „erinnerter Jesus“ mit dem nachösterlichen Titel ,Sohn Gottes" und mit Minimierung der magisch-rituellen Elemente deutlich an Kontur. In unmissverständlicher Deutlichkeit macht die Redaktionsgeschichte klar: Der magische Krankenheiler ist der Sohn Gottes.

120. Wolter, 2019, S. 148.

121. Lk 11,20; Mt 12,28.

122. Wolter, 2019, S. 121.

123. Heil, 2009, S. 93.

124. Vgl. Mk 3,22 parr.

ARYS, 18, 2020 [281-308] ISSN 1575-166x 


\section{Abiürzungen}

Eb. = Scholl, R. (Hrsg.) (2002). Der Papyrus Ebers. Die größte Buchrolle zur Heilkunde Ägyptens. Schriften aus der Universitätsbibliothek Leipzig 7. Leipzig: Universität Leipzig.

PapyCol = Merkelbach, R. und Totti, M. (Hrsg.) (1991). Abrasax. Ausgewählte Papyri Religiösen und Magischen Inhalts (PapyCol 17.1+2). Opladen: Westdeutscher Verlag.

P.Köln = Gronewald et al. (Hrsg.) (1987). Kölner Papyri Band 6. Sonderreihe Papyrologica Coloniensia 6. Opladen: Westdt. Verlag.

PGM = Preisendanz, K. (Hrsg.) (2001). Papyri Graecae Magicae = Die griechischen Zauberpapyri (Bd. 1-2). München: Saur.

RA = Contenau, G. und Dhorme, E. (Hrsg.). 1956. Revue d'Assyriologie et d'Archéologie Orientale, 50.2, S. 86-94.

$\mathrm{RGG}^{4}=$ Betz, H.-D. et al. (Hrsg.) (1998-2007). Religion in Geschichte und Gegenwart. Handwörterbuch für Theologie und Religionswissenschaft (Bd. 1-8). Tübingen: Mohr Siebeck.

\section{LITERATUR}

Alkier, S. und Weissenrieder, A. (Hrsg.) (2013). Miracles Revisited. New Testament Miracle Stories and their Concepts of Reality. SBR 2. Berlin: De Gruyter.

Becker, M. (2002). Wunder und Wundertäter im frührabbinischen Judentum. Studien zum Phänomen und seiner Überlieferung im Horizont von Magie und Dämonismus. WUNT 2, 144. Tübingen: Mohr Siebeck.

Belliger, A. und Krieger, D.J. (Hrsg.) (2013). Ritualtheorien. Ein einführendes Handbuch. Wiesbaden: Springer (5. Auflage).

Berger, K. (1995). Theologiegeschichte des Urchristentums: Theologie des Neuen Testaments. Tübingen: Francke (2. Auflage).

Bremmer, J.N. (2017). Maidens, Magic and Martyrs in Early Christianity. Collected Essays I. WUNT 379. Tübingen: Mohr Siebeck.

Brubaker, R. und Cooper, F. (2000). Beyond "Identity" Theory and Society, 29.1, S. 1-47.

Busch, P. (2006). Magie in neutestamentlicher Zeit. FRLANT 2018. Göttingen: Vandenhoeck und Ruprecht.

DeMarris, R.E. (2015). Ritualforschung: Eine Bereicherung für die neutestamentliche Wissenschaft. ZNT, 35, S. 31-42.

Douglas, M. (1974). Ritual, Tabu und Körpersymbolik. Sozialanthropologische Studien in Industriegesellschaft und Stammeskultur. Frankfurt/Main: S. Fischer.

Douglas, M. (2013). Ritual, Reinheit und Gefährdung. In Belliger und Krieger, 2013, S. 230261. 
Ebner, M. und Heininger, B. (2015). Exegese des Neuen Testaments. Paderborn: Schöningh (3. Auflage).

Eitrem, S. (1966). Some Notes on the Demonology in the New Testament. Uppsala: Almquist \& Wiksells (2. Auflage).

Eve, E. (2002). The Jewish Context of Jesus' Miracles. JSNT Supplement Series 231. New York, London: Sheffield Academic Press.

Frankfurter, D. (2002). Review: The Religious Context of Early Christianity: A Guide to Graeco-Roman Religions by Hans-Josef Klauck. The Journal of Religion, 82.3, S. 439-440.

Frazer, J.G. (2009). The Golden Bough. A Study in Magic and Religion. New York: Cosimo Classic (1922).

Frenschkowski, M. (2016). Magie im antiken Christentum. Eine Studie zur Alten Kirche und ihrem Umfeld. SAC 7. Stuttgart: Anton Hiersemann.

Gerstenberger, E.S. (1980). Der bittende Mensch. Bittritual und Klagelied des Einzelnen im Alten Testament. WMANT 51. Neukirchen-Vluyn: Neukirchener Verlag.

Heil, C. (2009). Evangelium als Gattung. Erzähl- und Spruchevangelium. In Schmeller, 2009, S. 63- 94.

Heimbrock, H.-G. (1994). Magie, Alltagsreligion und die Heilkraft des Glaubens. Etappen und Probleme theologischer und kulturwissenschaftlicher Magiediskussion. In Heimbrock und Streib, 1994, S. 17-59.

Heimbrock, H.-G. und Streib, H. (Hrsg.) (1994). Magie: Katastrophenreligion und Kritik des Glaubens: Eine theologische und religionstheoretische Kontroverse um die Kraft des Wortes. Innen \& Außen 1. Kampen: Kok Pharos.

Hellholm, D. et al. (2011). Ablution, Initiation, and Baptism. Waschungen, Initiation und Taufe. BZNW 176. Berlin und Leiden: Brill.

Kahl, W. (2013). New Testament Healing Narratives and the Category of Numinous Power. In Alkier und Weissenrieder, 2013, S. 337-350.

Kamlah, J., Schäfer, R. und Witte, M. (Hrsg.) (2017). Zauber und Magie im antiken Palästina und in seiner Umwelt. Kolloquium des Deutschen Vereins zur Erforschung Palästinas vom 14. bis 16. November 2014 in Mainz. Wiesbaden: Harrassowitz.

Kämmerer, T.R. (Hrsg.) (2007). Studien zu Ritual und Sozialgeschichte im Alten Orient. Tartuer Symposien 1998-2004. BZAW 374. Berlin und New York: De Gruyter.

Kremer, R. (2016). Glaube und Aberglaube. Wie aus Religion und Superstition ein Gegensatz wurde. Marburg: Tectum.

Kollmann, B. (2011). Neutestamentliche Wundergeschichten. Biblisch-theologische Zugänge und Impulse für die Praxis. Stuttgart: Kohlhammer (3. Auflage).

Mastrocinque, A. (2005). From Jewish Magic to Gnosticism. STAC 24. Tübingen: Mohr Siebeck.

McCauley, R.N. und Lawson, E.T. (2002). Bringing Ritual to Mind. Psychological Foundations of Cultural Forms. Cambridge: Cambridge University Press.

Meerson, M. und Schäfer, P. (Hrsg.) (2014). Toledot Yeshu: The Life Story of Jesus. TSAJ 159. Tübingen: Mohr Siebeck. 
Meyer, M. und Mirecki, P. (Hrsg.) (1995). Ancient Magic and Ritual Power. RGRW 129. Leiden: Brill.

Otto, B.-C. (2009). Magie. Rezeptions- und diskursgeschichtliche Analysen von der Antike bis zur Neuzeit. RGVV 57. Heidelberg: De Gruyter.

Paganini, S. (2017). Ein Gesetz zum Schutz der korrekten Kulthandlungen. Zauberei, Magie und andere verbotene Praktiken in Dtn 18,9-14. In Kamlah, Schäfer und Witte, 2017, S. 309-342.

Pietsch, M. (2017). Der Prophet als Magier. Magie und Ritual in den Elischaerzählungen. In Kamlah, Schäfer und Witte, 2017, S. 343-380.

Reed, A.Y. (2018). Jewish-Christianity and the History of Judaism. TSAJ 171. Tübingen: Mohr Siebeck.

Reimer, A.M. (2002). Miracle and Magic. A Study in the Acts of the Apostles and the Life of Apollonius of Tyana. JSNT Suppl. Series 235. New York: Sheffield Academic Press.

Reventlow, H.G. (2007). Krankheit - ein Makel an heiliger Vollkommenheit. Das Urteil altisraelitischer Priester in Leviticus 13 in seinem Kontext. In Kämmerer, 2007, SS. 275-290.

Ricks, S.D. (1995). The Magician as Outsider in the Hebrew Bible and the New Testament. In Meyer und Mirecki, 1995, S. 131-143.

Risto, U., Day, J., Roitto, R. und DeMaris, R. (Hrsg.) (2018). The Oxford Handbook of Early Christian Ritual. Oxford: Oxford University Press.

Sax, W., Quack, J. und Weinhold, J. (Hrsg.) (2010). The Problem of Ritual Efficiacy. Oxford: Oxford University Press.

Schmeller, T. (Hrsg.) (2009). Historiographie und Biographie im Neuen Testament und seiner Umwelt. Göttingen: Vandenhoeck \& Ruprecht.

Schmitt, R. (2017). Magie und rituelles Heilen im Alten Testament. In Kamlah, Schäfer und Witte, 2017, S. 183-197.

Smith, M. (1978). Jesus the Magician. London: Gollancz.

Stegemann, E.W. und Stegemann, E. (1997). Urchristliche Sozialgeschichte. Die Anfänge im Judentum und die Christusgemeinden in der mediterranen Welt. Stuttgart: Kohlhammer.

Swartz, M.D. (2018). The Mechanics of Providence. The Working of Ancient Jewish Magic and Mysticism. TSAJ 172. Tübingen: Mohr Siebeck.

Theißen, G. (2010). Jesus and his Followers as Healers: Symbolic Healing in Early Christianity. In Sax, Quack und Weinhold, 2010, SS. 45-66.

Theißen, G. und Merz, A. (2011). Der historische Jesus. Ein Lehrbuch. Göttingen: Vandenhoeck und Ruprecht (4. Auflage).

Theißen, G. (2017). Veränderungspräsenz und Tabubruch. Die Ritualdynamik urchristlicher Sakramente. BVB 30. Münster: LitVerlag.

Theißen, G. (2018). Ritual and Healing. In Risto et al., 2018, S. 445-461.

Uro, R. (2016). Ritual and Christian Beginnings. A Socio-Cognitive Analysis. Oxford: Oxford University Press.

Weber, M. (1982). „Die drei reinen Typen der legitimen Herrschaft.“ In Winckelmann, 1982, S. $475-488$. 
Winckelmann, J. (Hrsg.) (1982). Gesammelte Aufsätze zur Wissenschaftslehre. Tübingen: Mohr Siebeck (5. Auflage).

Wolter, M. (2019). Jesus von Nazareth. Theologische Bibliothek 6. Göttingen: Vandenhoeck \& Ruprecht. 